$$
N^{G^{3}} 3^{5^{2}}
$$

\title{
Combined Interpolation Scheme for Transition and Noble Metals $t$
}
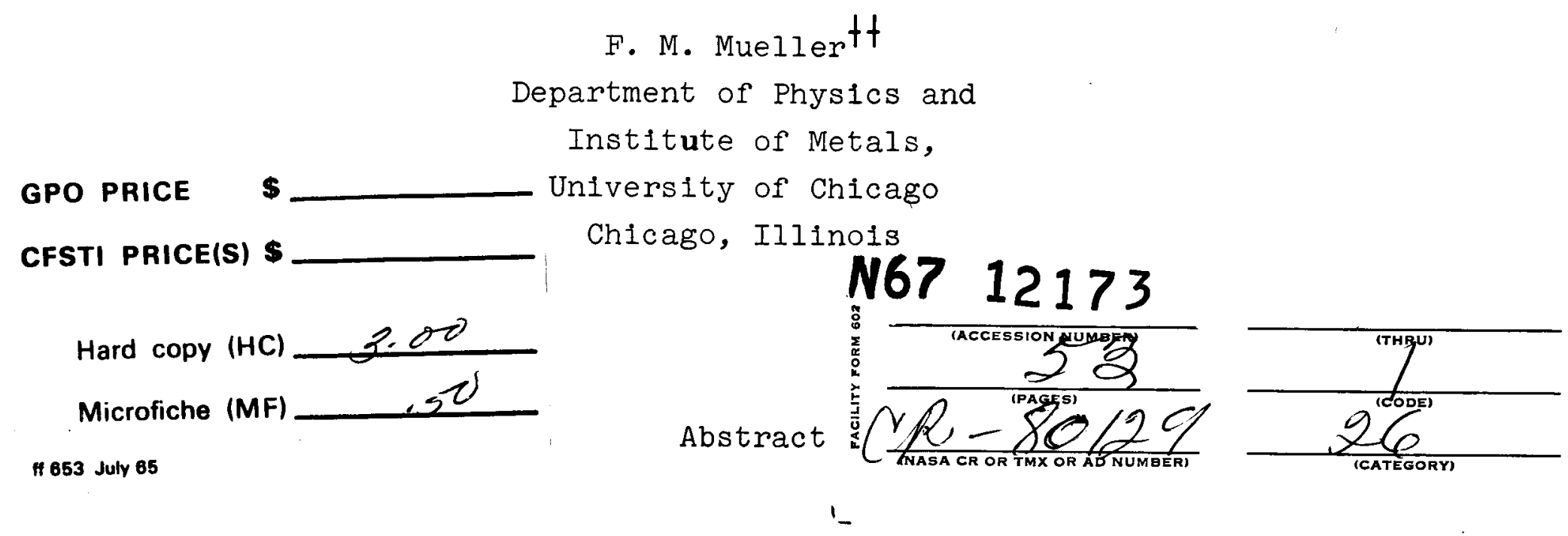

A combined interpolation scheme is presented for overlapping s-p conduction and $d$ bands. The $d$ bands alone are treated by the tight-binding method. The s-p conduction bands alone are treated by the pseudopotential method. It is shown that there are two important interactions between the bands which are called hybridization and orthogonalization; previous attempts to construct combined interpolation schemes included only the former interaction. By fitting the energy bands of $\mathrm{Cu}$ obtained by Segall and Burdick in first-principles calculations, we show that for the d bands alone the two-center approximation is valid to high accuracy. The rms error in the interpolated energy values for the fit $^{\prime} \mathrm{d}$ bands and the lowest conduction band is less than $0.1 \mathrm{eV}$ in both cases throughout the Brillouin zone. The relation of this scheme 
to resonance theories is indicated, and possible applications of the method are discussed.

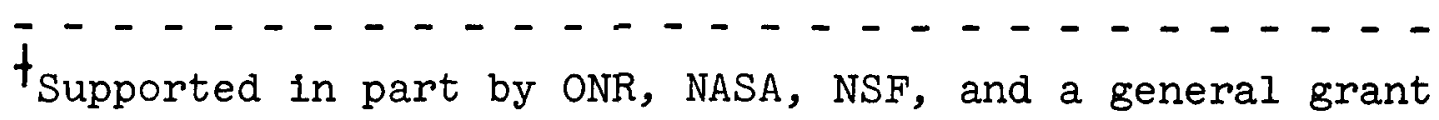
to ISM from ARPA.

ttGeneral Electric Fellow (1964-1965). 


\section{Introduction}

Calculations of the energy bands of solids can be classifled into two broad groups: first-princlple calculations which directly solve a given one-electron crystal wave equation, or interpolative calculations which describe the bands in terms of a minimal basis set and corresponding, disposable parameters. In the first group we include Orthogonalized Plane Wave (OPW) 1,2 and Augmented Plane Wave (APW) ${ }^{3}$ methods as well as the Green's function method.,5 In the second group are the atomic orbital scheme of Slater and Koster ${ }^{6}$ and the semi-empirical approaches based on pseudopotentials?,8

One of the first approaches to the band structure of solids was the LCAO or tight-binding method. The condition for its validity is that the one-electron wave functions be highly locallzed around each atomic core, with small overlap onto adjacent atoms. This condition is usually well met by the valence states of the rare gas solids and ionic crystals. In particular, it has. been used to calculate the valence band structure of $\mathrm{KCl}^{9}$ which roughly corresponds to $3 p$ atomic wave functions localized on the $\mathrm{Cl}^{-}$Lons.

The valence bands of the alkal1 halides furnish a simple example of the utility of an abstract approach. Howland considers separately the cases where the bands are derived from orbitals of $3 p\left(\mathrm{Cl}^{-}\right)$alone (a $3 \times 3$ secular equation), and where the basis set contains $3 \mathrm{~s}\left(\mathrm{Cl}^{-}\right), 3 \mathrm{p}\left(\mathrm{K}^{+}\right)$and $3 \mathrm{~s}\left(\mathrm{~K}^{+}\right)$orbitals also (an $8 \times 8$ secular equation). Energies from these two band structures ${ }^{10}$ are 
Iisted in Table I. The forms of the bands are seen to be similar to within $10 \%$, but the widths differ by a factor of 1.9 . Hence, a good description of the halide valence bands can be given in terms of only two "effective" overlap integrals (ppo) and $(\mathrm{pp} \pi)$. The "effective" overlap integrals turn out to have the same sign and ratio as those calculated from $3 p$ halide atomic orbitals, but are only about half as large. The "effective" basis functions are similar to Wannier functions, which are more localized and hence exhibit smaller overlap.

In view of the successful reduction of the actual bands derived using eight basis functions to an abstract model using only three basis functions and two shape parameters, one may now carry the process of abstraction one step further and determine the shape parameters directly from experiment. This has been done for CsBr from optical data; the measured parameters seem to be only $2 / 3$ of those calculated from eight basis functions?.11 This indicates that a further reduction in calculated band width would be obtained from a complete set of (exact) Wannier basis functions. From this example we conclude that an abstract scheme may actually yield better agreement with experiment than do first principles calculations. It also establishes a procedure for obtaining matrix elements between abstract basis functions (in this case Wannier functions), although the explicit determination of these functions is not required and may be inconvenient in practice. 
The formal treatment given in Section II closely parallels the OPW method. However, calculation of the $3 d$ states requires a combined tight-binding and plane wave approach. 15 such an approach is cumbersome if carried out rigorously. In section III we show that existing APW calculations justify representation of the $d$ bands alone by the Slater-Koster method. In Section IV, the s-p conduction band states are discussed including the effects of orthogonality to the d bands. In Section $V$ we treat s-d hybridization, and derive a parametric representation for the s-d potential terms.

At first sight, it might appear that treatment of the $s-d$ Interactions could be facilitated by use of group theory. Along certain lines of the Brillouin zone, symmetrical combinations of plane wave conduction band states can be formed which will be orthogonal to most of the d band states. The symmetry employed Is that of the group of each symmetry line. This approach does indeed simplify the treatment of $s-d$ hybridization effects along the (100), (110) and (111) axes. We have found, however, that for general $k$ values, this approach alone makes it very difficult to parameterize s-d interactions.

For this reason we have found it necessary to introduce a much stronger ansatz which is not consistent with group theory. However, the ansatz is valid to a good approximation, and therein lies its suitability for reducing the complexity of the parameterized representation. We assume that prior to hybridization with the lower plane waves the radial d wave functions in a given 
atomic cell are the same for all $|\underline{k}|$ and are independent of band index $n$. Thus the d states are regarded as part of the spherically symmetric atomic core. We justify our 1sotropic model for d states by direct comparison with APW band calculations.

The interpolation scheme developed here determines $E_{n}(\underline{k})$ throughout the Brillouin zone. Near points of high symmetry $\underline{k}_{\alpha}$ one can also expand $E_{n}(\underline{k})$ in powers of $\underline{k}-\underline{k}_{\alpha}$. This approach, usually called $\underline{k} \underline{p}$ perturbation theory, furnishes relations between our parameterized interactions and sheds light on their analytic character. It is alscussed elsewhere. 16

II. General Theory.

To be specific, we consider in this paper only monatomic fcc metals such as $\mathrm{N} 1$ and $\mathrm{Cu}$, although our results could easily be extended to monatomic bcc transition metals as well. The basis states are chosen as follows. To describe the $d$ bands, five states are required. These are taken to be proportional to $x y, x z, y z, x^{2}-y^{2}$, and $3 z^{2}-r^{2}$, which form a conventent representation for the angular dependence of tight-binding $d$ states in a cubic lattice. The lowest conduction bands in the positive 1/48th primitive section of the Brillouin zone (see F1g. 1) can be described using the four OPW's which are degenerate at the point $W$ in the empty lattice. These are labelled 
by their princlpal plane wave components $\left(\underline{k}+\underline{K}_{1}\right)$, where the reciprocal lattice vectors $\underline{K}_{i}$ are

$$
\begin{array}{ll}
\underline{K}_{0}=(0,0,0) & \underline{K}_{2}=(0, \bar{I}, 0) \\
\underline{K}_{1}=\left(\frac{\bar{I}}{2}, \frac{\bar{I}}{2}, \bar{I}\right) & \underline{K}_{3}=\left(\frac{\bar{I}}{2}, \bar{I} \frac{\bar{I}}{2}, \bar{I}\right)
\end{array}
$$

in units of $2 \pi / a$, and where barring means the negative.

Using these basis states, our $9 \times 9$ Hamiltonian will have the block form:

$$
5 \quad\left(\begin{array}{c:c}
5 & 4 \\
d-d & d-c \\
\hdashline c-d & c-c
\end{array}\right)
$$

In (2.2) d and $c$ stand for $d$ band and conduction band states, respectively. At a general point, $\underline{k}$, of the Brillouin zone all the matrix elements in (2.2) are non-zero.

We assume throughout that our basis functions are orthogonal, so that our secular equation has the form

$$
\operatorname{det}\left|H_{i j}-E \delta_{i j}\right|=0
$$

The assumption of orthogonality is essential if the abstract representation is to achieve the simplicity desired. We mention here brlefly reasons for hoping that a simple parametric representation of the matrix elements of (2.3) is feasible.

For the d bands we have the work of Fletcher and Wohlfarth ${ }^{17}$ who neglected non-orthogonality terms between d orbitals on dif- 
ferent atoms. We will show that the form of the d bands alone, AS given by APW calculations, is very close to that obtained by Fletcher and Wohlfarth.

For the conduction bands we find it necessary, in the spirit of the OPW and pseudopotential methods, to introduce energydependent orthogonality terms. These occur in the c-c block, and their analytic form is determined by our ansatz of d isotropy. Because the width of the $d$ band is comparable to the difference in energy between the conduction bands and the $d$ bands, these terms are no longer accurately proportional to $\left(E-E_{d}\right)$, as in the OPW method. Also less appropriate, near d-c crossover points, is the pseudopotential approximation $\mathrm{E} \rightarrow \overline{\mathrm{E}}_{\mathrm{c}}$, where $\overline{\mathrm{E}}_{\mathrm{c}}$ is a free-electron energy. 7,8

Our procedure for establishing each term in the secular equation is to make the general ansatz of $\mathrm{d}$ isotropy and then to determine the radial behavior of each term as a function of $\underline{k}$ by direct comparison with energy bands obtained by APW calculations. involving much larger secular equations. The full significance of this procedure will be treated in our summary. We find that the approximations to the orthogonality terms mentioned in the preceding paragraph often produce only small errors, and that for many purposes a simpler representation is adequate. Nevertheless, the full matrix form represents the most consistent and logically correct development of the isotropy ansatz, and hence forms the basis of our exposition. Our ultimate criterion for 
the successful reduction of the large secular equation--as well as our justification for the parameterization of the various terms in the Hamiltonian--will be the same one used in developing pseudopotentials, viz., success in obtaining $E_{n}(\underline{k})$ itself for the bands $\mathrm{n}$ of interest.

III. Tight Binding Representation of d Bands.

The tight-binding approximation may be formulated in several different ways. One may retain or neglect non-orthogonality terms between nearest neighbor basis functions. When the non-orthogonality terms are eliminated by unitary transformation of the tightbinding basis functions, the new basis functions are called Wannier or Lbwdin functions. Although this transformation does not alter the atomic symmetry of the basis functions and considerably simplifies the secular equation, it is difficult to carry out accurately. Another variation consists of neglecting three-centered integrals and non-orthogonality terms. This simplification, called the twocenter approximation, leads to substantial reductions in the number of overlap parameters.

Neglecting the s-p conduction bands, Fietcher and Wohlfarth ${ }^{17}$ have calculated the band structure of $\mathrm{N1}$ using the two-center approximation. They obtain a $5 \times 5$ secular equation in terms of certain nearest nelghbor overlap integrals. These they calculate using wave functions and a potential dertved by Hartree and Hartree 18 for $\mathrm{Cu}^{+}$. Although FW calculate six parameters, these are exactly 
equivalent to linear combinations of three two-center parameters. The relations satisfled by the six FW parameters in the two-center approximation ${ }^{6}$ are:

$$
\begin{aligned}
& A_{1}=-\frac{1}{4}(d d \sigma+d d \delta) \\
& A_{2}=\frac{1}{2}(d d \pi+d d \delta) \\
& A_{3}=\frac{1}{2}(d d \pi-d d \delta) \\
& A_{4}= \\
& A_{5}=-\frac{1}{4}\left(\frac{3}{4} d d \sigma+d d \pi+\frac{2}{4} d d \delta\right) \\
& A_{6}=-\frac{3}{8}(d d \sigma-d d \delta)
\end{aligned}
$$

Using our combined Interpolation scheme, we have fitted the $d$ bands and conduction bands of $\mathrm{Cu}$ as calculated by the APW method. 19 Inftially, we treated the general parameters such as A in (3.1) as Independent. Our best values for the first nelghbor parameters can be used to determine the three two-center, nearest neighbor overlap integrals. The result, in terms of our parameters $P_{1}$ (see appendix $B)$, is

$$
\begin{aligned}
& d d \sigma=\left(P_{3}-\frac{1}{\sqrt{3}} P_{6}\right) ;\left(P_{7}-\sqrt{3} P_{6}\right) ; \frac{1}{2}\left(3 P_{3}-P_{7}\right) \\
& d d \pi=\left(P_{4}+P_{5}\right) ; P_{8} \\
& \operatorname{dd} \delta=\left(P_{3}+\sqrt{3} P_{6}\right) ; \frac{1}{2}\left(3 P_{7}-P_{3}\right) ;\left(P_{4}-P_{5}\right) ;\left(\frac{1}{\sqrt{3}} P_{6}+P_{7}\right)
\end{aligned}
$$


We compare the fitted left-hand sides of (3.2) - (3.4) with the values for the right-hand sides derived from (3.1) in Table II.: It appears that the two-center approximation is valid to about $6 \%$. The corresponding differences in a band energy levels are at most 0.004 Ry., with one exception.

The exceptionally large three-center contribution is represented by the zero of energy of $\Gamma_{25}$, relative to $\Gamma_{12}$, which is described in the notation of ref. 6 by $E_{11}(000)-E_{55}(000)=0.008 \mathrm{Ry}$. (Note that this is not equivalent to $\Gamma_{12}-\Gamma_{25} \cdot$ ) We have therefore included this correction explicitly in the Hamiltonian, but have otherwise made the two-center approximation.

The detailed evaluation of the $E$ or $P$ parameters 1 s given in Appendix A. Briefly, the method consists of obtaining a set of linear equations in the parameters through the natural factorization of the secular equation at Brillouin zone symmetry points and along symmetry lines. The matrix elements of the $d$ part of the Hamiltonian can now be obtained from Slater and Kosteris Table III (reproduced in Appendix A), providing that the zero of energy parameters are $\underline{d}_{0}$ for the triplet degeneracy at $\Gamma$ and $\underline{d}_{0}+\gamma$ for the doublet degeneracy where $\gamma=0.008 \mathrm{Ry}$. In $\mathrm{Cu}$.

We conclude by discussing the four second nelghbor E parameters. These were included in the determination of parameters discussed in Appendix A, but they were found to be so small (<0.001 Ry.) as to have a negligible effect on the band structure. For this reason, we have not included them in our final scheme. Altogether, this leaves the $5 \times 5$ d-d block parameterized in terms of $\underline{d}_{0}, \gamma$, 
$\underline{\mathrm{dd}} \sigma, \underline{\mathrm{dd}} \pi$ and $\underline{\mathrm{dd}} \delta$.

IV. Representation of Conduction Bands.

At first glance, the calculated conduction bands of transition and noble metals appear to be nearly free electron in character. However, on closer examination, one sees that there are marked deviations in the lowest conduction band, depending on whether one Is above or below the $\mathrm{d}$ bands. Moreover, the Bragg splittings at the symmetry points $X$ and $I$ are much larger than those found in "simple" metals such as Al.

To 1llustrate these deviations, we have plotted in Fig. 2 Segall's results ${ }^{20}$ for the first and second conduction bands in $\mathrm{Al}$ and the corresponding bands in $\mathrm{Cu}^{21}$ It is clear that the $\mathrm{d}$ bands are responsible for several differences in behavior which we analyze in two limits. In the first, the conduction band is close to a $d$ band of the same symmetry. Here hybridization has taken place-the $\mathrm{d}$ band and conduction band are split equally above and below the point of crossover in the absence of interaction. We call this splitting the direct $c-d$ effect.

In the second limit, the conduction band is well above the $d$ bands. There the conduction band is shifted above the free electron band by a roughly constant amount. Note, however, that the shifts are different for the varlous symmetry directions (100), (110), and (111), being in the ratio (1:2:1) respectively. (Note that this is also the ratio of the number of $d$ bands whose group representation 1 s the same as the lowest conduction band for these three symmetry directions.) Thus, if we wish to incorporate these 
deviations into the secular equation, we must have terms that are both angularly as well as radially dependent.

The hybridizing and repulsive effects are indicated for the energy bands ${ }^{19}$ of $\mathrm{Cu}$ along the (110), (100) and (111) symmetry a:es in Fig. $3(\mathrm{a})-(\mathrm{c})$, respectively. Our task now $1 \mathrm{~s}$ to construct an analytic representation of these effects. Procedures for obtaining the hybridizing terms will be discussed in the following section. To determine the repulsive terms, we assume that in terms of pseudoplane wave states $|\underline{k}\rangle$ the conduction band state $\left|\varphi_{\underline{k}}\right\rangle$ has the form

$$
\left|\phi_{\underline{k}}\right\rangle=\left[|\underline{k}\rangle-\sum_{n} M_{d n}(\underline{k})|d n\rangle\right] c_{\underline{k}}^{-1}
$$

where the normalizing factor $c_{k}$ is given by

$$
\left|c_{\underline{k}}\right|^{2}=1-\sum_{n}\left|M_{d n}(\underline{k})\right|^{2}
$$

Our assumption of a isotropy can be used to determine the orthogonality coefficients $\mathrm{M}_{\mathrm{dn}}(\underline{\mathrm{k}})$ as follows. Let a d basis function be written as

$$
|\mathrm{dn}\rangle=\phi_{\mathrm{dn}}(\underline{r})=\mathrm{C}_{\mathrm{n}} \mathrm{F}_{\mathrm{n}}^{\mathrm{d}}(\mathrm{x} / \mathrm{r}, \mathrm{y} / \mathrm{r}, \mathrm{z} / \mathrm{r}) \mathrm{g}(\mathrm{r})
$$

where $C_{n}$ is the normalizing factor for the cubic harmonic $F_{n} d$. Then

$$
\begin{aligned}
M_{\mathrm{dn}}(\underline{\mathrm{k}}) & =\left\langle\phi_{\mathrm{dn}} \mid \underline{\mathrm{k}}\right\rangle \\
& =\mathrm{C}_{\mathrm{n}} \mathrm{F}_{\mathrm{d}}\left(\mathrm{k}_{\mathrm{x}} / \mathrm{k}, \mathrm{k}_{\mathrm{y}} / \mathrm{k}, \mathrm{k}_{\mathrm{z}} / \mathrm{k}\right) \mathrm{f}(\mathrm{k})
\end{aligned}
$$

Because of $d$ isotropy $f$ is a function only of $k$, and not of $k$. The explicity parameterization of $f(k)$ is given below. 
Matrix elements in the $4 \times 4$ conduction block are given by

$$
\left.\phi_{\underline{\underline{k}}}|\mathrm{H}| \phi_{\underline{\underline{k}^{\prime}}}\right\rangle=\mathrm{C}_{\underline{\underline{k}}}^{-1} \mathrm{C}_{\underline{k}^{\prime}}^{-1}[I+I I+I I I+I V]
$$

where in units with $h^{2} / 2 m=1$,

$$
I=\left(\underline{k}^{2}+V_{0}\right) \delta_{\underline{k k^{\prime}}}+V_{\underline{k}-\underline{k}^{\prime}}\left(1-\delta_{\underline{k k^{\prime}}}\right) \text {. }
$$

The terms $V_{\underline{k}-\underline{k}}$ represent the sum of the matrix elements of the crystal potential $V$ and the repulsive potential $V_{R c}$ between reciprocal lattice plane waves ${ }^{7,8}$. We let $K_{1}$ denote all the (111) and (200) reciprocal lattice vectors. For $K_{f}$ larger than $K_{1}$, we can apply the general cancellation arguments ${ }^{8}$ and set $v\left(K_{f}\right)=0$. This leaves us with two conduction band pseudopotential parameters $\mathrm{V}_{111}$ and $\mathrm{V}_{200^{\circ}}$

One finds that $V_{111}$ and $V_{200}$ alone produce band gaps comparable to those of the "simple" metals. In Fig. 2, we have labelled this part of the splitting of $\mathrm{L}_{1}$ and $\mathrm{L}_{2}$, by $2 \mathrm{~V}_{111}$.

The $d-d$ orthogonality terms in (4.5) are

$$
I V=\Sigma_{n^{\prime}} C_{n} C_{n^{\prime}} F_{n}^{d}(\underline{k}) F_{n^{\prime}}^{d}\left(\underline{k}^{\prime}\right) f(k) f\left(k^{\prime}\right) H_{d n}, d n^{\prime}
$$

where $\mathrm{H}_{\mathrm{dn}}$, dn' is the approprlate matrix element between the tightbinding $d$ states contained in the $d-d$ block. The cross terms can be shown to have the form

$$
\begin{gathered}
I I+I I I=V-2(I V) \\
V=-\sum_{n} \sum_{\prime^{\prime}} F_{n}(\underline{k}) F_{n^{\prime}}\left(\underline{k}^{\prime}\right)\left[\underline{C}_{\underline{k}} g(k) f\left(k^{\prime}\right)+\underline{C}_{\underline{k}^{\prime}} g\left(k^{\prime}\right) f(k)\right]
\end{gathered}
$$


where $g(k)$ is the hybridizing form factor discussed in section 5 . For our matrix element we finaliy have

$$
\left\langle\phi_{\underline{k}}|H| \phi_{\underline{k}^{s}}\right\rangle=C_{\underline{k}}^{-I} C_{\underline{k}^{\prime}}^{-I}[I+V-I V]
$$

We must also consider the terms

$$
E\left\langle\Phi_{\underline{k}} \mid \phi_{\underline{k^{\prime}}}\right\rangle=E\left[f_{\underline{k k^{\prime}}}-\left(1-\delta_{\underline{k k^{\prime}}}\right) O T\right]
$$

where OT represents positive orthogonality terms. The normalization factors in (4.11) make the diagonal coefficient of $E$ unity. The off-diagonal OT are inconvenlent, and if retalned would complicate the machine solution of the secular equation considerably.

In the pseudopotential approximation to the OPW method the terms $E(O T)$ are grouped with the terms $-E_{d}(O T)$, which are represented by IV in (4.10). One then makes the replacement $E \Rightarrow \overline{E_{c}}$ (an average plane wave energy) and assumes that the $d$ bands are narrow $\left(E_{d}-\overline{E_{d}}\right)$. The resulting OT are proportioned to $\overline{E_{c}}-\overline{E_{d}}$ and are manifestly invariant to a change in the zero of energy.

In our case, we have found it simpler to proceed as follows. The conduction band width $\delta E_{c}$ is large; in fact $\delta E_{c}>\left(\overline{E_{c}}-\overline{E_{d}}\right)$. However, one can consider the OT in two limits: near s-d crossovers and near the (200) and (111) Bragg scatterling planes. In the former case direct calculation shows that very good results are obtained by neglecting the OT altogether compared to the hybridization terms. Th1s is not surprising, for the hybridization 
terms are large in magnitude and have a larger effect because of the quas1-degeneracy of the $d$ bands and conduction bands.

On the other hand, near the Bragg scattering planes the nondiagonal OT can be made small by a proper cholce of the zero of energy. We have found that setting $E(X)=x^{2}+y_{c}=0$ gives good results for conduction band states at $I, X$ and $W$.

[ By neglecting $E(\underline{k})+V_{0}$ at $I$ and $W$, we in effect, absorb small OT into our pseudopotential parameters. Again direct calculation shows that the error incurred is small.]

From d 1sotropy, one can see that as $k \rightarrow 0, f(k)$ is of order $k^{2}$. Thus a convenient form for $f(k)$ is

$$
f(k)=A j_{2}\left(k R_{0}\right)
$$

where $f_{2}(x)$ is the second spherical Bessel function that is obtained in the OPW method by expanding plane waves in terms of Legendre polynomials. To improve convergence $f(k)$ is cut of $f$ beyond 1ts second node. The value of $L R \circ 1$ s 2.9 for $\mathrm{Cu}$ and $\mathrm{A}=1.3$. In Fig. 4, we show $f(k)$ explicltiy. The procedure used to determine $A$ and $R_{0}$ is described in Appendix $B$.

The orthogonal1ty terms are responsible for the large Bragg splittings at $X$ and $I$, which are asymmetric with respect to the free electron energies at these polnt. For example, neglect the effects of $s-d$ hybridization (section 5) and consider the conduction band structure assoclated with only the two lowest plane waves near $X$ or $I\left(X_{4}\right.$, and $X_{1}$, or $I_{2}$, and $\left.I_{1}\right)$. Here the lowest two bands have 
wave vector $\underline{k}$ and $-\underline{k}$. We extract from the secular equation these degenerate levels giving a $2 \times 2$ determinant:

$$
\text { aet. }\left|\begin{array}{cc}
\mathrm{K}^{2}+\mathrm{a}-\lambda & \mathrm{a}+\mathrm{V}_{2 \mathrm{~K}} \\
+\mathrm{V}_{0} & \mathrm{~K}^{2}+\mathrm{a}-\lambda \\
\mathrm{a}+\mathrm{V}_{2 \mathrm{~K}} & +\mathrm{v}_{0}
\end{array}\right|=0
$$

where $\lambda$ is the elgenvalue, $K^{2}$ is the kinet1c energy, $V_{2 K}$ is $V_{111}$ or $V_{200}$ for $L$ and $X$ respectively, and $a$ is the term derived from orthogonality. Solving this simple determinant yields:

$$
\lambda=\left(K^{2}+a\right) \pm\left(V_{2 K}+a\right)+V_{0}
$$

Hence we see that for the lower (odd symmetry) eigenvalue the effect of orthogonality is identically zero, whereas for the higher (even symmetry) level $2 a$ is added. The asymmetry of the repulsive term a in the two eigenvalues shows that marriage of tight-binding and plane wave techniques requires orthogonalization of the plane wave states to the d states.

Another approach which reveals a relation between the tightbinding diagonal block and the plane wave block, and which is independent of $s-d$ hybridization, is based on $\underline{k} \cdot \underline{p}$ perturbation theory near $\mathrm{X}$ or $\mathrm{L}$. One can then show that orthogonalization terms in the latter block are required to balance overlap (finite band width) terms in the a block. 
V. s-d Hybridization.

In this section we consider the matrix elements of the Hamiltonian in the off-diagonal blocks $c-d$ and $d-c$. Although the conduction basis states given by equation (4.1) have been orthogonalized to the $d$ basis states, c-d matrix elements $w i l 1$ st1ll be non-zero in general. In fact near c-d cross-overs, where the orthogonality terms are small, the hybridization terms alone separate $c$ and $d$ terms belonging to the same irreducible representations.

It is consistent within our isotropic approximation to represent the crystal potential as a superposition of spherically symmetric atomic potentials. Just as second-neighbor d-d overlap was found to be small compared to nearest neighbor overlap, so we neglect nearest nelghbor overlap in computing the hybrid1zation term. Thus we regard the mixing as derived from interactions in a spherically symmetric central cell. Using (4.1) we have

$$
\left\langle\operatorname{dn}\left|H^{\prime}\right| \Phi_{\underline{k}}\right\rangle=C_{\underline{k}}^{-1}\left[\left\langle\operatorname{dn}\left|H^{\prime}\right| k\right\rangle-H_{d n, d^{\prime}}^{\prime} M_{d n}(\underline{k})\right]
$$

Strictly speaking $H^{\prime}$ in (5.1) includes both the crystal potential and the repulsive terms arising from orthogonalization of the plane wave $|\underline{k}\rangle$ to the $s$ and $p$ core states. With complete isotropy the latter would vanish in determining the matrix element (5.1) with d states. In any case we are not concerned here with 
the details of (5.1). For our purposes it is sufficient to write

$$
\left\langle d_{n}\left|H^{\prime}\right| \varphi_{k}\right\rangle=F_{n}^{d}(k) g(k)
$$

where $g(k)$ is an isotropic hybridizing form factor. With the absorption of the normalization factor, $C_{k}$, the definition (5.2) is consistent with (4.9).

To be completely consistent one should represent the first term on the right hand side of (5.1) by a form factor $g^{\prime}(k)$ and determine $g(k)$ from $g^{\prime}(k)$ using (5.1). In practice the effects of hybridizing are dominated by the first term, and it is not profitable to carry through this separation.

Again $1 t$ can be shown that, as $k \rightarrow 0$, we have $g(k) \rightarrow k^{2}$. Thus for small $\mathrm{k}$ we set

$$
g(k)=B \dot{j}_{2}\left(k R_{1}\right)
$$

with $\mathrm{LR}_{1}^{\prime}=2.9$ and $\mathrm{B}=13.8 \mathrm{eV}$ in $\mathrm{Cu}$. We show $\mathrm{g}(\mathrm{k})$, including a linear cutoff at large $k$, in Fig. 5 .

For a given crystal potential and an assumed $d$ wave function (e.g., taken from the free atom) we could evaluate (5.1). However, to obtain agreement with APW calculations is would then be necessary to include the effects of higher plane waves on the d states. For reasons discussed in our introduction and summary, this is just what we wish to avold. The form factor $g(k)$ introduced here in- 
cludes these effects consistently both in equation (5.2) and in equation (4.9).

In Appendix $B$ we discuss in detail the method used to determine $g(k)$. It is similar to that described in section 3 for determining the $d$ band parameters. There we considered only d band states of symmetry different from the conduction band. Here we use d band states along symmetry lines of symmetry types the same as those of the crossing conduction bands. Between these $d$ and conduction bands we have both hybridization and orthogonality terms. The effects of the former are larger near s-d crossoversi [As discussed in the preceding section, we found it convenient to neglect certain orthogonality contributions to the matrix elements in the region, because of its tight-binding character. Had we retalned these terms, and introduced other (less accurate) approximations instead, the orthogonality terms could have been forced into the form $\left(E-E_{d}\right)\left|M_{d n}(k)\right|^{2}$. In this form one can see. explicitly how to separate the hybridization splittings from the orthogonality terms, by assuming that the latter vanish near $s-d$ crossovers and using the construction of Fig. 3.] Indeed we find that near $s-d$ crossovers the hybridization terms actually dominate so strongly as to yleld an unambiguous separation. On the other hand, near the zone faces where Bragg scattering takes place, the orthogonality terms dominate and the hybridization form factor can be determined by iteration. Another method for separating hybridization from orthogonality 
terms near the zone faces relies on the $k \cdot p$ perturbation theory ${ }^{16}$ near $X$ and $L$. This method is extremely accurate in the immediate vicinity of symmetry points, but the interaction method described In Appendix B yields more general results throughout the Brillouin zone. These are also quite accurate, and appear to approach the limitations inherent in the ansatz of $d$ isotropy.

VI. Evaluation and Simple Applications

To fit the $d$ bands of a monatomic fec metal using our method, certain parameters must be specified. For the conduction bands alone there are two parameters $-V_{111}$ and $V_{200}$. The position and shape of the $d$ bands alone are flxed by five parameters--do (the $d$-band energy relative to the conduction bands), ddo, dd $\pi$, dds, and the three-center parameter $\gamma$. Finally the $s-d$ interactions are specified by $A, B, R_{0}$ and $R_{1}$ in equations (4.12) and (5.3). For convenience these eleven parameters are listed in Table VI. Values are given there which fit the energy bands of $\mathrm{Cu}$ as determined by Burdick from an $\boldsymbol{k}$-independent potential using the APW method ${ }^{19}$, as well those calculated by Segall using an $l$-dependent potential in the multiple scattering formulation ${ }^{20-22}$.

By examining the table one notices several differences between Burdick's and Segall's calculations which arise from the $l$-dependent potential used by the latter. Segall's d wave functions are more extended than are Burdick's. This is reflected in the d band overlap 
parameters, which are about 30\% greater for Segall's bands than for Burdick's. The orthogonality and hybridization strengths ( $A$ and $B$, respectively) are $20 \%$ and $5 \%$ greater, respectively. (We believe that this indicates that the two effects cannot be combined into one.) The pseudopotential parameters $V_{11 I}$ and $V_{200}$ are also changed, and are larger for Segall's bands than for Burdlck's, reflecting the fact that the $R$-dependent potential leads to greater $c-d$ differences, and somewhat stronger $c-c$ interactions. The scale factors $R_{0}$ and $R_{1}$ change by $5 \%$ and $16 \%$, respectively, which again suggests the independence of the hybridizing and orthogonality terms.

The accuracy of the scheme can be tested in several ways. The overall rms deviation between our values and those of Burdick for the first six bands ( $5 \mathrm{~d}$ bands and lowest conduction band) at 89 points of the Brillouin zone is $0.08 \mathrm{eV}$. The values at $\Gamma, \mathrm{X}, \mathrm{L}$ and W are compared in Table IV; for these points the rms deviation is $0.07 \mathrm{ev}$. Similar results are obtained in fitting segall's bands. The most critical test of the parameterization is obtained by comparing the rms deviation of the set of 20 points used to determine relations among the parameters fitting Erirdick's bands with that of the set of 40 points not used; the two values are 0.07 and $0.08 \mathrm{ev}$, respectively. It can be seen that within statistical uncertainties the deviations are identical. Moreover, the uncertainties in varIous APW $C u$ energy levels (apart from those assoclated with $d_{0}$, the position of the d bands relative to the conduction bands) are still 
larger than these rms deviations, so that a more accurate fit might not be significant. Finally the levels at $X, I, K$ and $W$ in the second conduction band are reproduced with an accuracy comparable to that of the first principles calculations.

As a simple application of our scheme we have calculated the density of states $g(E)$ in the d band region both with and without mixing of the $d$ bands and conduction bands. In the calculations preserted below we have constructed histigram densities of states based on the lowest 6 eigenvalies of our 9th order secular equations at 4500 randomly selected points in 1/48th of the Brillouin zone. With an energy interval of 0.0025 ryd this gives an average $\wedge$ $n_{1}$, of 300 and an rms fluctiation $i n r_{1}$ of about $5 \%$. If one so desires (as we did in the comparisons kelow) the same random selection can be used in each set of samples.

We have considered three band structures related to Burdick's $\mathrm{Cu}$ hands. The first, given in Fig. 6 , corresponds to the d bands alone. Tris case (which has been calonated bef ne ${ }^{23}$ ) was used as a cheok or the technique. We set that this is quite similar to the results obtained previously, exoept that our Fig. 6 has more structure due to the seven times larger random sample.

In the other two cases (shown in Figs. 7 and 8) we have added the s-d interactions to the bare d-band structume of Fig. 6 . The band parameters for case 2 are taken fiom the fit to Burdick's calculation discussed in preceding section. Case 3 has the same 
band parameters, except that the position of the conduction bands has been changed with respect to the $d$ band complex. Here we have increased the zero of energy $\phi_{0}$ of the $\alpha$ bands relative to the conduction bands, leaving the remaining parameters unchanged. Case 2 (Fig. 7) has $\alpha_{0}=+5.84 \mathrm{eV}$. (copper-like), whereas case 3 (Fig. 8) takes $d_{0}=+7.20 \mathrm{eV}$ (nickel-like).

By comparing Fig. 8 with Fig. 7 we see that shifting the conduction bands relative to the d bands leaves the density of states throughout most of the d band region almost unchanged. This is what one would expect from a rigid band model, neglecting s-d mixing altogether. Both the large peaks in the density of states near 0.3 and 0.4 ryd. and weaker peaks near 0.22 and 0.35 ryd. are little changed, indicating that they arise from states of almost pure d character.

In Fig. 7 and Fig. 8 we have indicated energies at the symmetry points $\Gamma, X, I$ and $W$. These produce Van Hove edges in the density of states. Other critical points are important, however, and these are not located at symmetry points. (E.g., the peak in the density of states near the top of the $d$ band, which is commonly supposed to explain the high specific heat of $N i$, is caused on the higher side by the $\mathrm{I}_{3}$ edge, and on the lower side by an unidentified critical point.) 
VII. Conclusions

The alm of this paper has been to develop a combined interpolation scheme which could reproduce the energy bands of transition and noble metals within $0.1 \mathrm{eV}$ and which would depend on the smallest possible number of parameters. In reaching our goal we have shown from APW calculations for $\mathrm{Cu}$ that

(1) only nearest neighbor interactions in the two-center approximations (plus one three-certer term) are required for the d bands;

(2) Only two pseudopotential parameters are required to describe the lowest conduction band;

(3) That conduction band-d bard interactions are of two kinds. The first, hybridization, is well known, but 1ts magnitude has been evaluated and it has been shown (apart from spherical harmonic factors) to be isotropic. The presence of the second interaction, a repulsive one arising from the requirement of orthogonalization of basis states, had not previously been recognized in AFW calculations;

(4) Both conduction band-d band Interactions are describable in terms of form factors. Although the factors are similar, they appear to be independent.

Some of the assumptions upon which our calculations are based have been discussed in several recent papers ${ }^{24,25}$. The d bands are regarded as a resonant level overlapping the conduction band, and 
the phase shift of the resonant level is introduced using scattering theory. These discussions are entirely formal, whereas our results demonstrate explicitly that an abstract approach can reproduce APW or multiple scattering calculations very accurately. Although previous formal discussions based on phase shifts have not been able to separate hybridizing and orthogonality terms, we have shown that both terms must be included to achieve high accuracy. A major unsolved problem for the resonance theories is how to incorporate five (rather than one) d resonance levels into the theory. We have avoided this problem at the outset and then have gone on to demonstrate (we belleve for the first time) the validity of the two-center approximation. A precise sense in which the resonance analogy is valid is analyzed in the following paper ${ }^{16}$.

Because of the simplicity and generality of the interpolation scheme, It should have wide applications to many problems in the quantum structure of materials containing overlapping conduction and d bands, fust as the simple pseudopotential method has successfully treated s- and p-band crystals. We mention only two applications which follow immediately from the method in its present form. Firstly one may determine parametric values to yleld very accurate fits to observed Ferml surfaces. Secondly the high speed of the method together with the natural character of the basis states (atomic d states or plane wave conduction states) makes calculation of direct interband optical spectra, including proper oscillator strengths, straightforward. We hope to return to these applications elsewhere. 


\section{ACKNOWLEDGEMENTS}

We wish to express our gratitude to Professors J. C. Phillips and M. H. Cohen for suggesting this problem. We are especially indebted to the former for his constant help and encouragement, without which this paper would never have appeared in its present form. In addition, we wish to thank Mr. J. Hermanson, and Drs. N. Ashcroft and D. Penn for useful conversations. Finally, we wish to acknowledge the excellent service given by the staff of the University of Chicago's Computation Center. 
APPENDIX A

In Table $\mathrm{V}$ are listed the twelve parameters given by the zeroth, first, and second neighbor general overlap integrals. There are two difficulties in determining these parameters from a given band structure calculation. First, the eigenvectors of an energy level are not, in general, wholly $d$, but have some hybridization with the conduction bands. Second, even in those levels which are most d-like, the energy level will depend on several of these parameters, rather than on one or two.

Both of these difficulties can be overcome by considering energy levels at points in the Brillouin zone of high symmetry. Then only one, or at most two, of the d levels will have a symmetry type 1dentical to some low conduction band, and only these d levels will hybrid1ze. The remaining levels at this point can be used to determine linear relations among the parameters. In addition, along symmetry lines, the secular equation conveniently factorizes into many relations among few parameters.

There is one more criterion for selecting relations among the parameters. The points and levels should be chosen so that the resultant statistical weight of each parameter is approximately the same. This results in equal errors in the parameters. Equality in statistical 
weight can be guaranteed if the points used are equally spaced along each symmetry line.

The evaluation of the parameters for copper was a simple matter since the energy bands had been calculated by Burdick at 89 points in the Brillouin zone and $2 / 3$ of these points were along symmetry lines. Thus, approximately 150 useful levels remained after combining with 5 d-levels, roughly half of which were unhybridized or non-degenerate levels. Of these we selected 48, so that each parameter was represented approximately five times. (Note that one of the two zeros of energy must appear in each equation.) These relations yield the overdetermined linear equations

$$
A_{i j} X_{j}=E_{i}
$$

where $A$ is the rectangular coefficient matrix, $E_{i}$ are the energy eigenvalues, and $\mathrm{x}_{j}$ are the parameters. (We sum on repeated indices.)

The $R \cdot M \cdot S$ deviation of the $X_{j}$ will be minimized if

$$
A_{i k} A_{i j} X_{j}=A_{i k} E_{i}
$$

calling $A_{i k} A_{i j}=C_{k j}$ and $A_{i k} E_{i}=D_{k}$, then $\subseteq$ is a symmetrical, non-singular, square matrix. 


\section{8}

The desired parameters are given by

$$
X_{j}=\left(C^{-1}\right)_{k j} D_{k}
$$

We can solve (A-3) using standard routines from a computer library such as SHARE.

In Table $\mathrm{V}$ we list these parameters, as well as the values calculated by Fletcher and Wolfarth. The similarity in values is remarkable, considering the divergent sources of the two columns.

By allowing a full three-centered treatment of the $d$ bands in copper, we have shown that an additional restriction to two-centered overlap integrals makes a negligible error in the band structure. Henceforth we shall calculate the $d$ bands in two-centered approximations except for the zero-of-energy parameter Table VI lists the $d$ band matrix elements as derived from slater-Koster ${ }^{6}$. 
APPENDIX B

The cholce of Bessel functions $j_{2}(k R)$ to parameterize the hybridization and orthogonality form factors is a natural one. The scale factor $\mathrm{R}$ represents an average of the values of $r$ for which the $c-d$ potential interactions or overlap (respectively) are largest. The best value of $R$, which should not change greatly from one transition metal to the next, are determined as described below.

For larger values of $k$ the replacement of a welghted value of $j_{2}$ (kp) over a range of $r$ by a local value $j_{2}$ (kR) will obviously be poor, because $j_{2}(k r)$ oscillates in sign. This effect is incorporated into the form factors by introducing a linear cutoff at large $k$. This cutoff plays a small role in our calculations, because of the nearly spherical shape of the fcc Brillouln zone. However, a much greater effect is expected when the Brillouin zone is more anisotropic, as for bcc crystals.

The "Iongest" radial symmetry dimension of the fcc zone appears to be $\Gamma \Sigma K X$. The lowest conduction band along $\mathrm{KX}$ is $\Sigma_{3}$, and when this band $1 \mathrm{~s}$ continued onto $\Sigma=\Gamma \mathrm{K}$, it becomes the second lowest conduction band. For this range of $k$ the cutoff effects are important. They can be separated by noting that the $\Sigma_{2}$ and $\Sigma_{3}$ d bands are symmetrical 
with respect to $\Gamma_{25}$ in the absence of interactions with the conduction bands. This symmetry holds well for $k$ between $\Gamma$ and $K / 2$ so that a linear cutoff was introduced between $K$ and $3 K / 2$.

W1th eleven parameters to be determined, it was necessary to develop simple schemes to find best values. Our scheme proceeded as follows. With the values of the d band parameters determined as described in Appendix A, $A, B, R_{0}$ and $R_{1}$ were determined approximately by solving $2 \times 2$ and $3 \times 3$ c-d interaction secular equations along $\Delta, \Lambda, \Sigma$ and $Z$. With these approximate values the rms deviation of the fit was determined to be about $0.2 \mathrm{eV}$. The parameters were varied by fixed increments and quadratic interpolation was used to minimize the rms deviations. This procedure converged rapidly ( 5 minutes on an IBM 7094 ) and uniquely to the values of the parameters quoted in Table III. 


\section{REFERENCES}

* Submitted in partial fulfillment of the requirements for the degree of Doctor of Philosophy at the University of Chicago.

1 C. Herring, Phys. Rev., 57, 1169 (1940).

2 T. O. Woodruff, Solid State Physics, 4, (Academic Press, New York, 1959).

3 J. C. Slater, Phys. Rev., 51, 846 (1937).

4 W. Kohn and N. Rostoker, Phys. Rev. 94, 1111 (1954).

5 F. S. Ham and B. Segall, Phys. Rev. 124, 1786 (1961).

6. J. C. Slater and G. F. Koster, Phys. Rev. 94, 1498 (1954).

7 J. C. Phillips and I. Kleinman, Phys. Rev. 116, 287 (1959).

8 M. H. Cohen and V. Heine, Phys. Rev. 122, 1821 (1961).

9 L. P. Howland, Phys. Rev. 109, 1927 (1958).

10 I. P. Bouckaert, R. Smoluchowski, and E. Wigner, Phys. Rev. 므, 58 (1936).

11 J. C. Phillips, Phys. Rev. 136, Al721 (1964).

12 Such a combined scheme was mentioned briefly by M. Saffren in reference 13 only for states at $X$ and $L$. Because he omitted the s-d orthogonality terms, Saffren concluded that such a scheme was not feasible. After completion of the bulk of this work a brlef note appeared (see ref. 14) purportedly discussing a combined interpolation scheme. Although no detalls are given, this scheme appears to be equivalent to Saffren's and to suffer from the same deficiencies. For example, we estimate that the 
rms errors incurred by omitting the orthogonality terms would be well in excess of $1.0 \mathrm{eV}$.

13 (see below)

14 L. Hodges and H. Ehrenreich, Physics Letters 16, 203 (1965).

15 J. C. Phillips, Phys. Rev. 133, Al020 (1964).

16 J. C. Phillips (to be published).

17 G. C. Fletcher and E. P. Wohlfarth, Phil. Mag. 42, 106 (1951);

G. C. Fletcher, Proc. Phys. Soc. (London) A, 65, 192 (1952).

18 D. R. Hartree and W. Hartree, Proc. Roy. Soc. A, 157, 490 (1936).

19 G. A. Burdick, Phys. Rev., 129, 138 (1963).

20 B. Segall, Phys. Rev. 124, 1797 (1961).

21 B. Segall, Phys. Rev. 125, 109 (1962).

22 We thank Dr. Segall for a list of his energy elgenvalues for both copper and aluminum.

23 G. F. Koster, Phys. Rev. 98, 901 (1955).

24 J. M. Ziman, Proc. Phys. Soc. 86, 337 (1965).

25 V. Heine, Phys. Rev. (companion paper).

13. The Fermi surface (W. A. Harrison and M. B. Webb, Editors, Wiley, New York, 1960), p. 341. 


\section{TABLE CAPTIONS}

Table I. Ratio of the energy differences of two calculations of the valence bond structure of $\mathrm{KCl}$ (see reference 9).

Table II. Comparison of our derived two-center parameters with those calculated by FW from $\mathrm{Cu}^{+} \mathrm{d}$ wave functions. The deviation of the various values from their mean is a measure of the validity of the two-center approximation.

Table III. Values of model parameters chosen for two copper band structures (ref. 19 and 20). The significance of differences between the parameters is discussed in section VI.

Table IV. Interpolated values of the lowest seven levels at $\Gamma$, $X, I$, and $W$ as obtained from the parametric values Iisted in Table III are compared with the calculated values of Burdick (ref. 19) and Segall (ref. 20). The fit is equally good in both cases. (All values are listed in rydbergs.)

Table $V$. The values of the Slater-Koster overlap parameters $E_{1}$ as obtained from fitting Burdick's band structure for $\mathrm{Cu}$ are shown in column 4. These are to be compared with the values listed in column 5 obtained from the two-center formulae of column 3. The values of ddo, 
$\mathrm{dd} \pi$, and dd $\delta$ were also obtained by fitting Burdick's $\mathrm{Cu}$ bands and are listed in Table III. The percentage differences listed in the last column provide an indication of the validity of the two-center approximation. Table VI. The matrix elements of the $d-d$ block in the two-center approximation. The symbol $x$ represents $k_{x} a / 2$ and similarly for $y$ and $z$. 


\section{TABLE I}

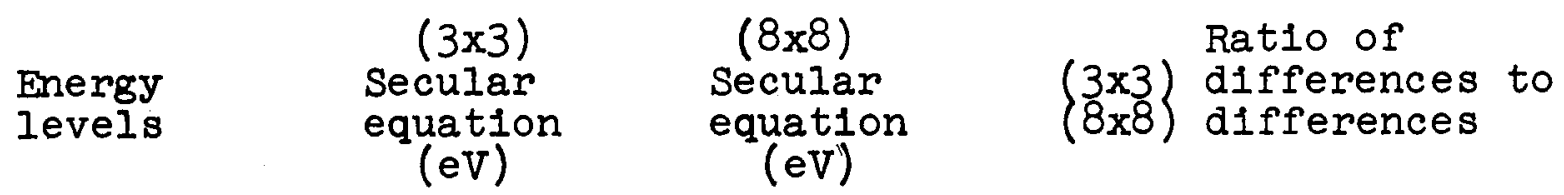

$$
\begin{aligned}
& \Gamma_{15^{-}}-x_{5^{\prime}} \\
& \Gamma_{15^{\prime}}-x_{4^{\prime}} \\
& x_{5^{\prime}}-x_{4^{\prime}} \\
& I_{3^{\prime}}-\Gamma_{15} \\
& \Gamma_{15^{\prime}}-I_{2} \\
& I_{3^{\prime}}-I_{2^{\prime}}
\end{aligned}
$$$$
0.56
$$$$
0.27
$$$$
2.07
$$$$
1.63
$$$$
0.98
$$$$
1.66
$$$$
1.07
$$$$
0.52
$$$$
2.05
$$$$
0.42
$$$$
0.24
$$$$
1.75
$$$$
2.44
$$$$
1.26
$$$$
1.93
$$

2.87

1.51

1.90

Mean Rat10 =

1.89 
TABLE II

\begin{tabular}{|c|c|c|c|c|}
\hline $\begin{array}{l}\text { Overlap } \\
\text { parameter }\end{array}$ & $\begin{array}{l}\text { Value derived } \\
(3.2)^{\text {from }}-(3.4)\end{array}$ & Mean & $\begin{array}{c}\text { RMS } \\
\text { Deviation \% }\end{array}$ & $\begin{array}{l}\text { Value } \\
\text { calculated } \\
\text { by FW }\end{array}$ \\
\hline \multirow[t]{3}{*}{$d d \sigma$} & $-0.348 \mathrm{eV}$ & $-0.348 \mathrm{eV}$ & $0.0 \%$ & $-0.338 \mathrm{eV}$ \\
\hline & $-0.348 \mathrm{eV}$ & & & \\
\hline & $-0.348 \mathrm{eV}$ & & & \\
\hline \multirow[t]{2}{*}{$\mathrm{dd} \pi$} & $+0.163 \mathrm{eV}$ & $+0.178 \mathrm{eV}$ & $8.4 \%$ & $+0.182 \mathrm{eV}$ \\
\hline & $+0.192 \mathrm{eV}$ & & & \\
\hline \multirow[t]{4}{*}{$d d \delta$} & $-0.0217 \mathrm{eV}$ & $-0.0213 e V$ & $2.7 \%$ & $-0.026 \mathrm{eV}$ \\
\hline & $-0.0217 \mathrm{eV}$ & & & \\
\hline & $-0.0204 \mathrm{eV}$ & & & \\
\hline & $-0.0217 \mathrm{eV}$ & & & \\
\hline
\end{tabular}


TABLE III

Parameter $\quad$ Burdick $\quad$ Segall

d Bands

\begin{tabular}{|c|c|c|}
\hline$d_{0}$ & $+5.84 \mathrm{eV}$ & $+4.95 \mathrm{eV}$ \\
\hline ddo & $-0.35 \mathrm{eV}$ & $-0.45 \mathrm{eV}$ \\
\hline $\mathrm{dd} \pi$ & $+0.18 \mathrm{eV}$ & $+0.24 \mathrm{eV}$ \\
\hline $\mathrm{dd} \delta$ & $-0.02 \mathrm{eV}$ & $-0.04 \mathrm{eV}$ \\
\hline$\gamma$ & $+0.08 \mathrm{eV}$ & $-0.01 \mathrm{eV}$ \\
\hline$v_{111}$ & $+0.07 \mathrm{eV}$ & $+0.26 \mathrm{eV}$ \\
\hline$v_{200}$ & $+0.46 \mathrm{eV}$ & $+0.55 \mathrm{eV}$ \\
\hline
\end{tabular}

Onthogonality

$A$
$L R_{0}$

1.29

1.59

2.88

3.03

Hybridization

B

$13.78 \mathrm{eV}$

$13.92 \mathrm{eV}$

L $R_{1}$

2.93

3.47

Errors

$\sqrt{\sigma_{i}^{2}}$

$0.06 \mathrm{eV}$

$0.08 \mathrm{eV}$ 


\section{8}

TABLE IV

\begin{tabular}{|c|c|c|c|c|}
\hline Level & Burdick point & $\begin{array}{c}\text { Interpolation } \\
\mathrm{fit}\end{array}$ & SegalI & $\begin{array}{l}\text { Int. } \\
\text { fit }\end{array}$ \\
\hline$\Gamma_{1}$ & -1.043 & -1.043 & -0.836 & -0.837 \\
\hline$\Gamma_{25^{\prime}}$ & -0.640 & -0.647 & -0.505 & -0.510 \\
\hline$\Gamma_{12}$ & -0.582 & -0.574 & -0.433 & -0.426 \\
\hline & & ' & & \\
\hline $\mathrm{X}_{1}$ & -0.776 & -0.776 & -0.666 & -0.667 \\
\hline$x_{3}$ & -0.739 & -0.740 & -0.630 & -0.634 \\
\hline$x_{2}$ & -0.540 & -0.535 & -0.383 & -0.376 \\
\hline$x_{5}$ & -0.527 & -0.534 & -0.366 & -0.371 \\
\hline$x_{4}$, & -0.235 & -0.243 & -0.024 & -0.032 \\
\hline$x_{1}$ & +0.152 & +0.145 & +0.389 & +0.401 \\
\hline $\mathrm{I}_{1}$ & -0.775 & -0.774 & -0.646 & -0.638 \\
\hline$I_{3}$ & -0.642 & -0.648 & -0.511 & -0.518 \\
\hline$I_{3}$ & -0.538 & -0.543 & -0.380 & -0.385 \\
\hline$I_{2}$, & -0.429 & -0.435 & -0.247 & -0.241 \\
\hline$I_{1}$ & -0.094 & -0.099 & +0.189 & +0.201 \\
\hline$w_{2}$ ' & -0.723 & -0.718 & -0.607 & -0.599 \\
\hline$w_{3}$ & -0.671 & -0.676 & -0.537 & -0.536 \\
\hline$w_{1}$ & -0.585 & -0.583 & -0.438 & -0.438 \\
\hline$W_{1}$, & -0.527 & -0.536 & -0.365 & -0.371 \\
\hline$w_{3}$ & +0.105 & +0.116 & +0.310 & +0.313 \\
\hline
\end{tabular}




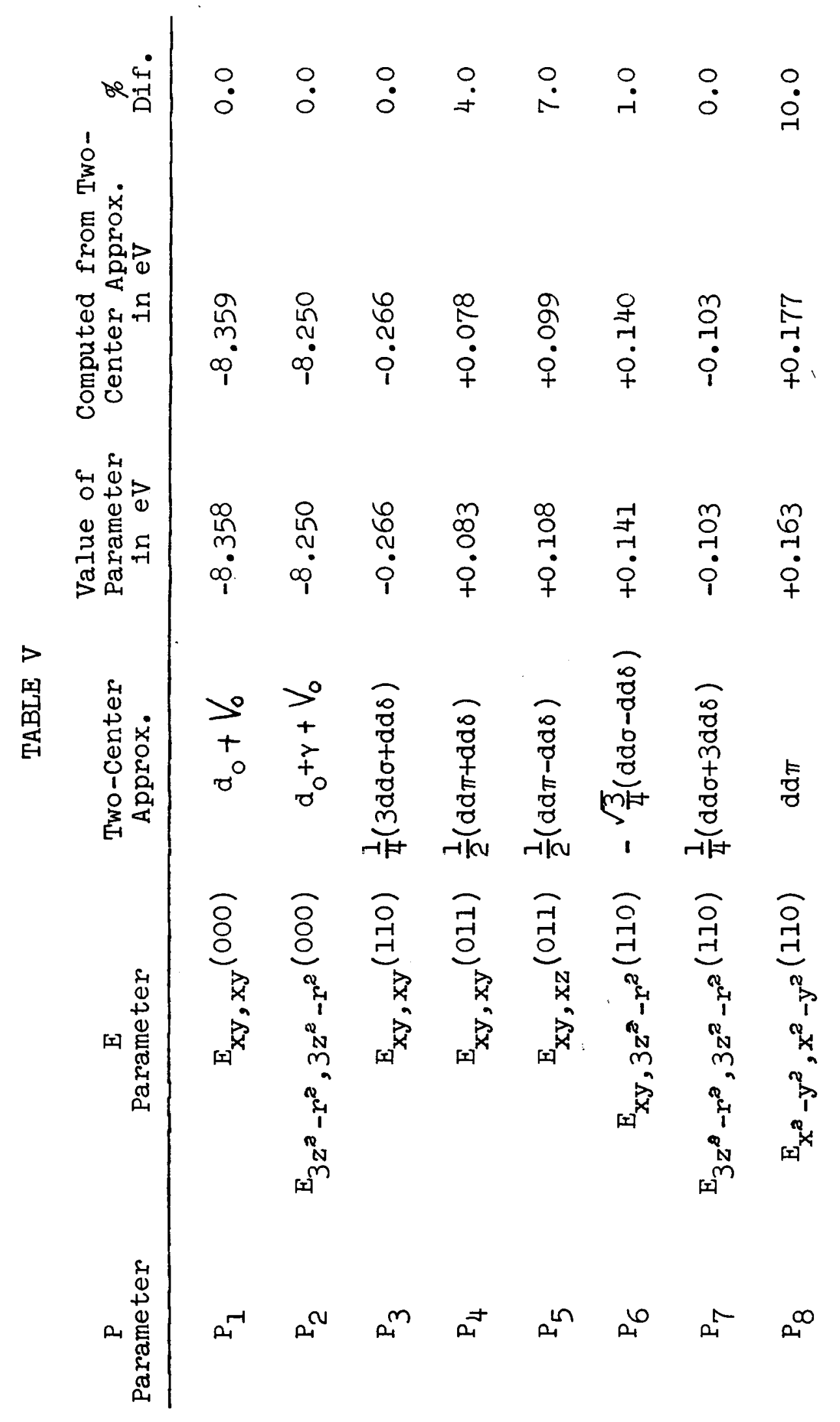




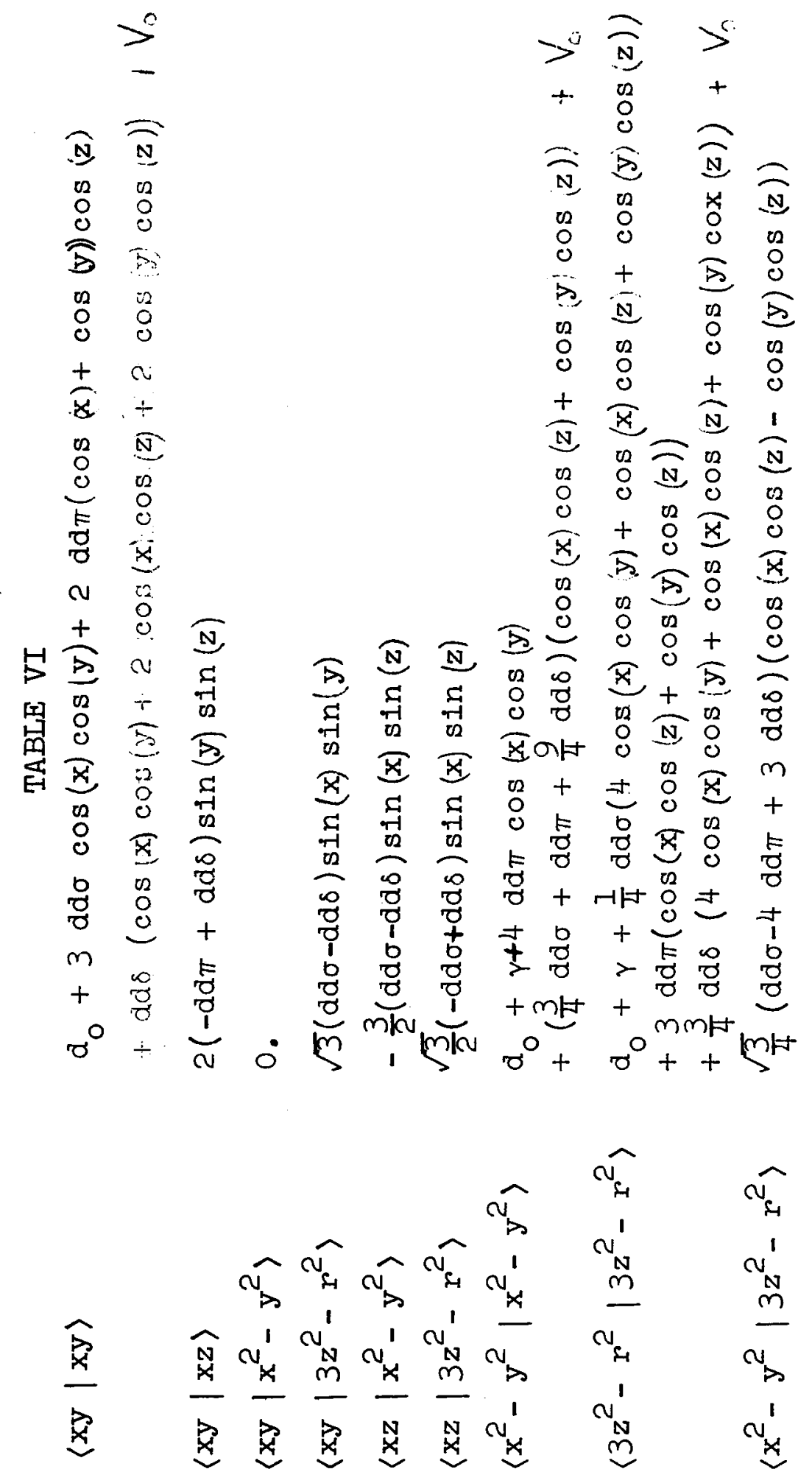




\section{1}

Figure Captions

1) The Brillouin zone of the f.c.c. lattice showing the $1 / 48$ th primitive wedge used in the calculations.

2) Schematic representation of the hybridization and repulsive effects between the $\mathrm{Cu} \mathrm{d}$ bands and the lowest two conduction bands aling the symmetry line $\Lambda$. Only the bands of $\Lambda_{1}$ symmetry are shown. The dashed lines show the unperturbed bands of Al or the tight-binding $\mathrm{d}$ band of $\mathrm{Cu}$ in the absence of these two effects. (The $A I$ bands have been scaled so that $\left(\frac{n^{2} L^{3}}{2 m}\right)_{a I}=$ $\left(\frac{n^{2} k^{a}}{2 m}\right)_{c u}$ at $\left.k=L_{.}\right)$

3) The energy levels of $\mathrm{Cu}$. The solid circles represent the calculated values of Burdick (reference 19) and the solid lines represent the interpolated bands obtained using the parameters Iisted in Table III. The small differences shown on the scale of the solid circles are genuine and can be ascribed to breakdown of the ansatz of $d$ isotropy.

4) The repulsive form factor $f(k)$ exhibits a maximum for $k$ near the Brillouin zone edges. The value of $f(k)$ shown here is taken from the parameters used to fit Burdick's band structure (see Table III).

5) The hybridization form factor $g(k)$. One can show that crystal symmetry requires that $g^{\prime}(k)=0$ for $\underline{\underline{k}}=\underline{I}$ and $\underline{x}$, where the prime indicates the directional derivative of $\mathrm{g}(\underline{\mathrm{k}})$ normal to the $\mathrm{L}$ or $\mathrm{X}$ face (respectively). Our assumption of $\mathrm{g}$ isotropy then suggests that if this is satisfied exactly $g$ should be flat for 
$\underline{k}$ between $\underline{I}$ and $\underline{X}$. We have chosen to ignore this cond1tion, because as shown here $g^{\prime}(X)$ is very nearly zero, and $g^{\prime}(L)$ is small. As a result $g$ has a simple analytic form. The linear cut-off discussed in Appendix $B$ begins at $4 \mathrm{ak} / \pi=$ 9.3 .

6) The density of $\mathrm{d}$ band states in $\mathrm{Cu}$ retaining the width due to the tight-binding interactions $d d \sigma$, $d d \pi$, dd $\delta$ but omitting the conduction bands as well as their interactions with the d bands. Five major peaks appear in the density of states, at 0.27 , $0.29,0.33,0.36$ and 0.39 ryd.

7) The density of $d$ band and conduction band states using the Burdick $d$ band parameters as in Fig. 6 , but including interactions with the conduction bands. In the $d$ band region the densities of states are qualitatively similar, but numerous quantitative differences are apparent. Thus the peak present in Fig. 6 at 0.27 ryd. has disappeared, while the 0.29 and 0.33 ryd. peaks of Fig. 6 have merged into one peak here at $0.31 \mathrm{ryd}$. The peak at $0.22 \mathrm{ryd}$ and the shoulder starting near $0.15 \mathrm{rgd}$. arise from those states near the bottom of the d band which hybridize strongly with the conduction band.

8) Here the conduction band has been shifted relative to the d band by $-1.4 \mathrm{eV}$. The effects on the density of states are very small, as can be seen by comparing with the density of states shown in Fig. 7. For many purposes this provides justification 
of the rigid band model of ten used to discuss the properties of transition metal alloys. 


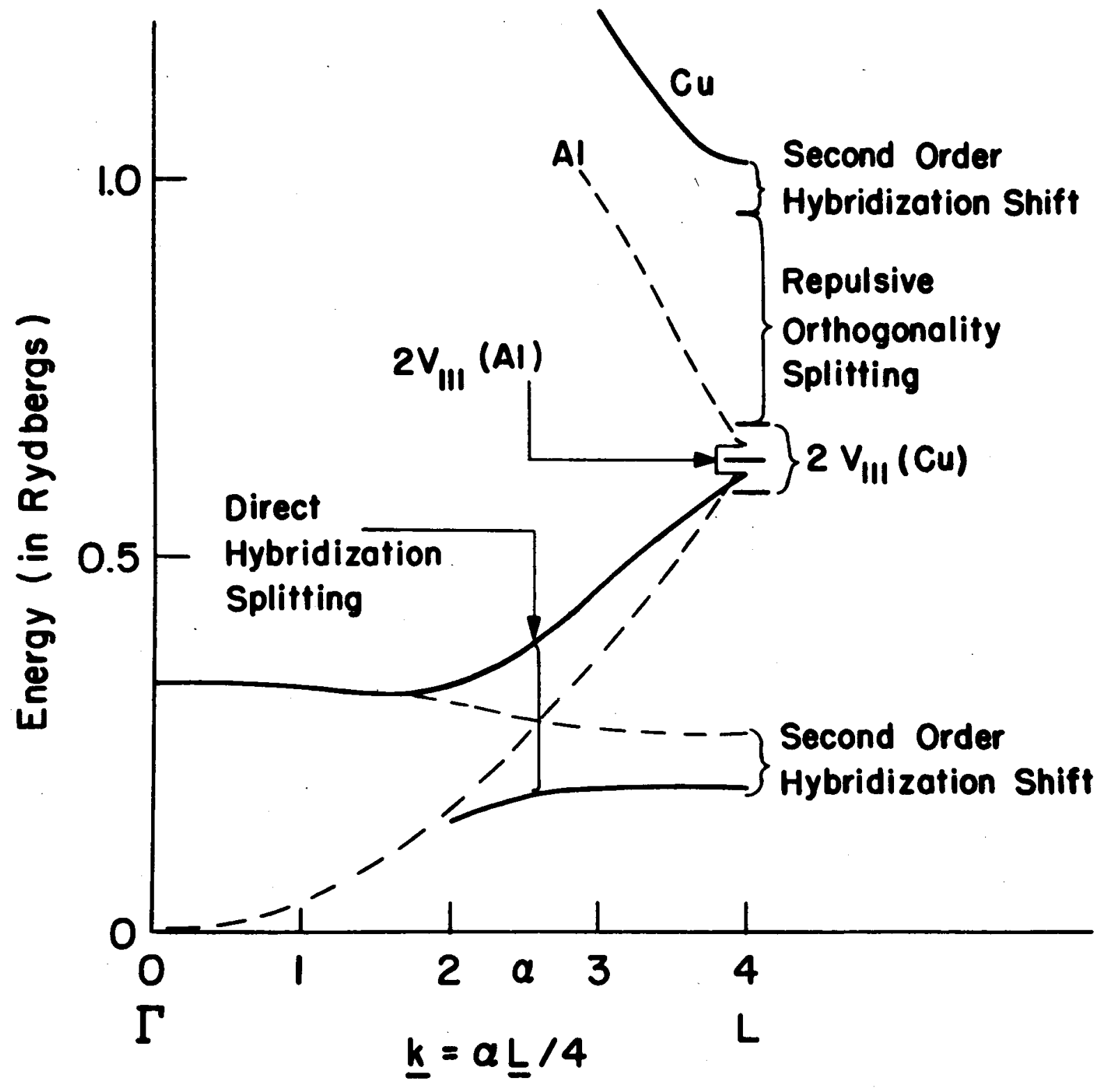

Fig. I 


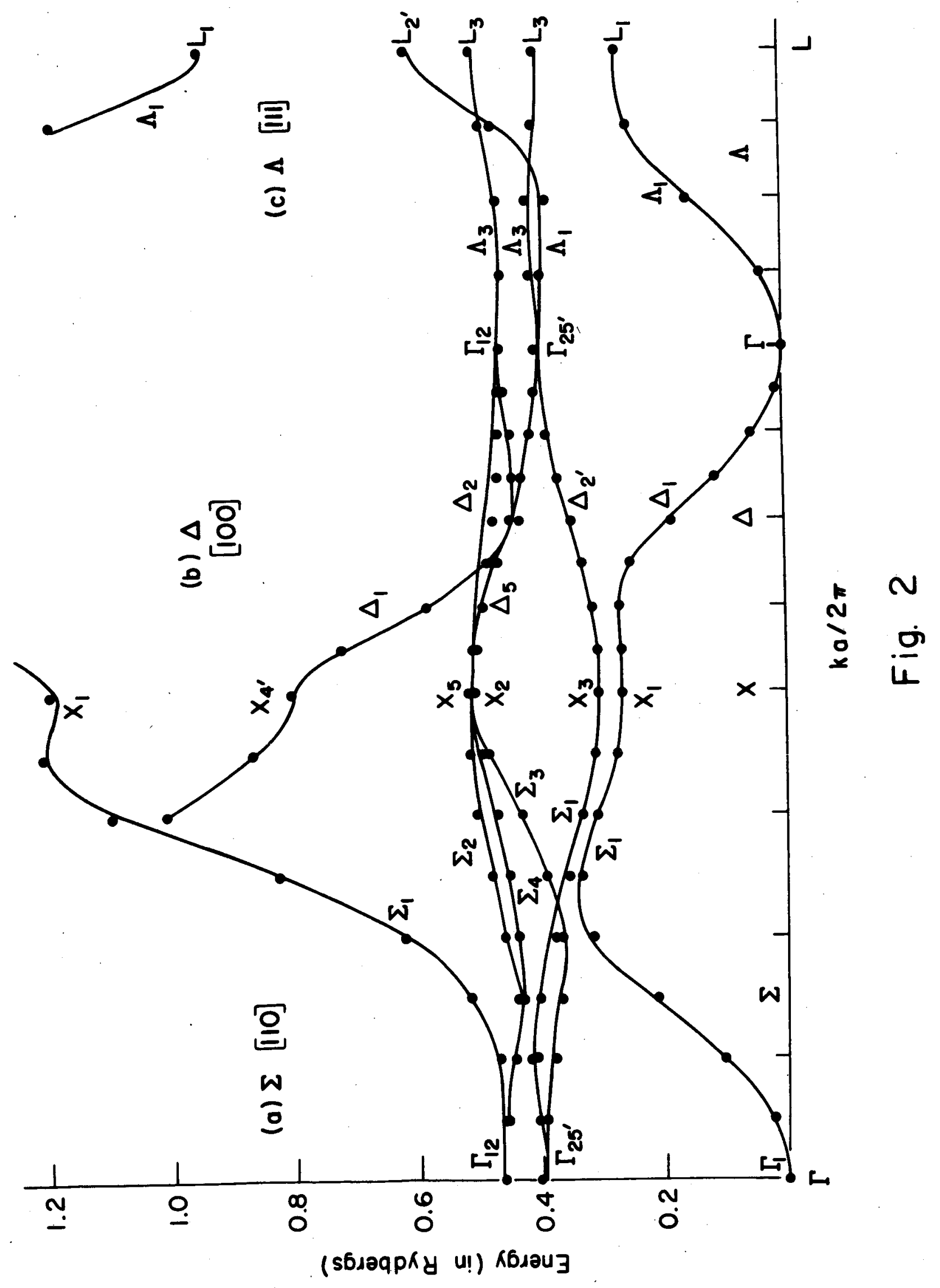




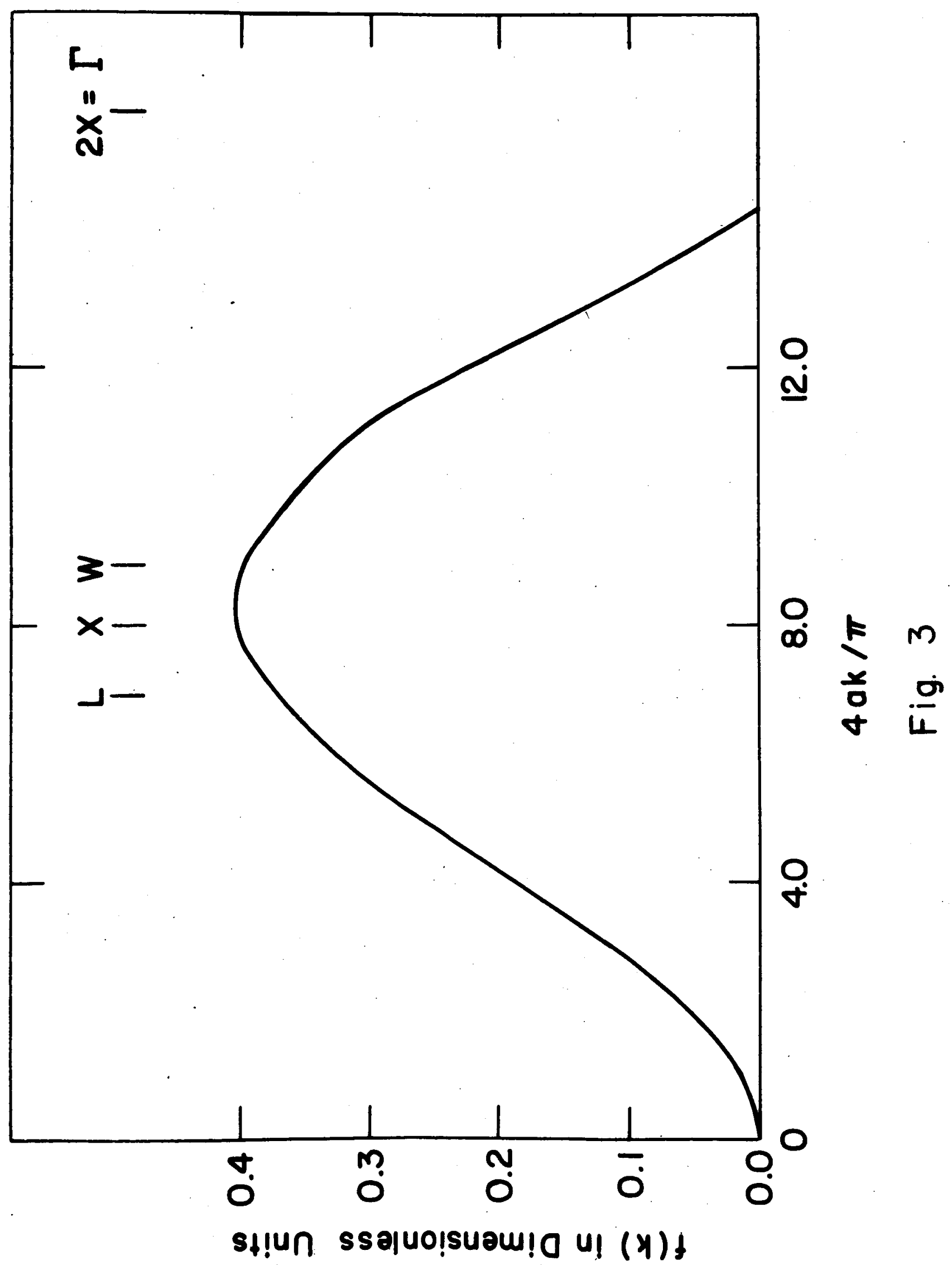




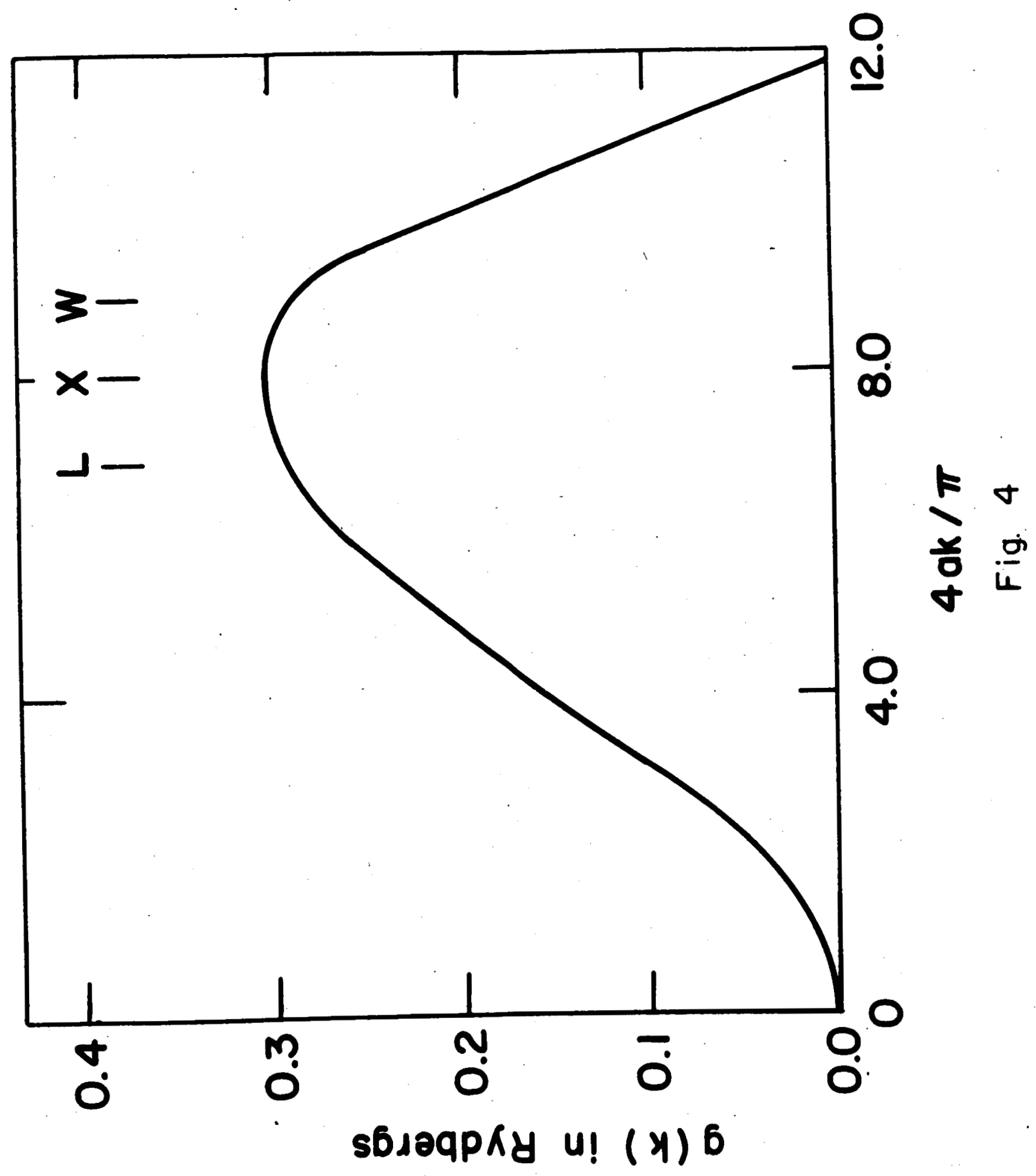




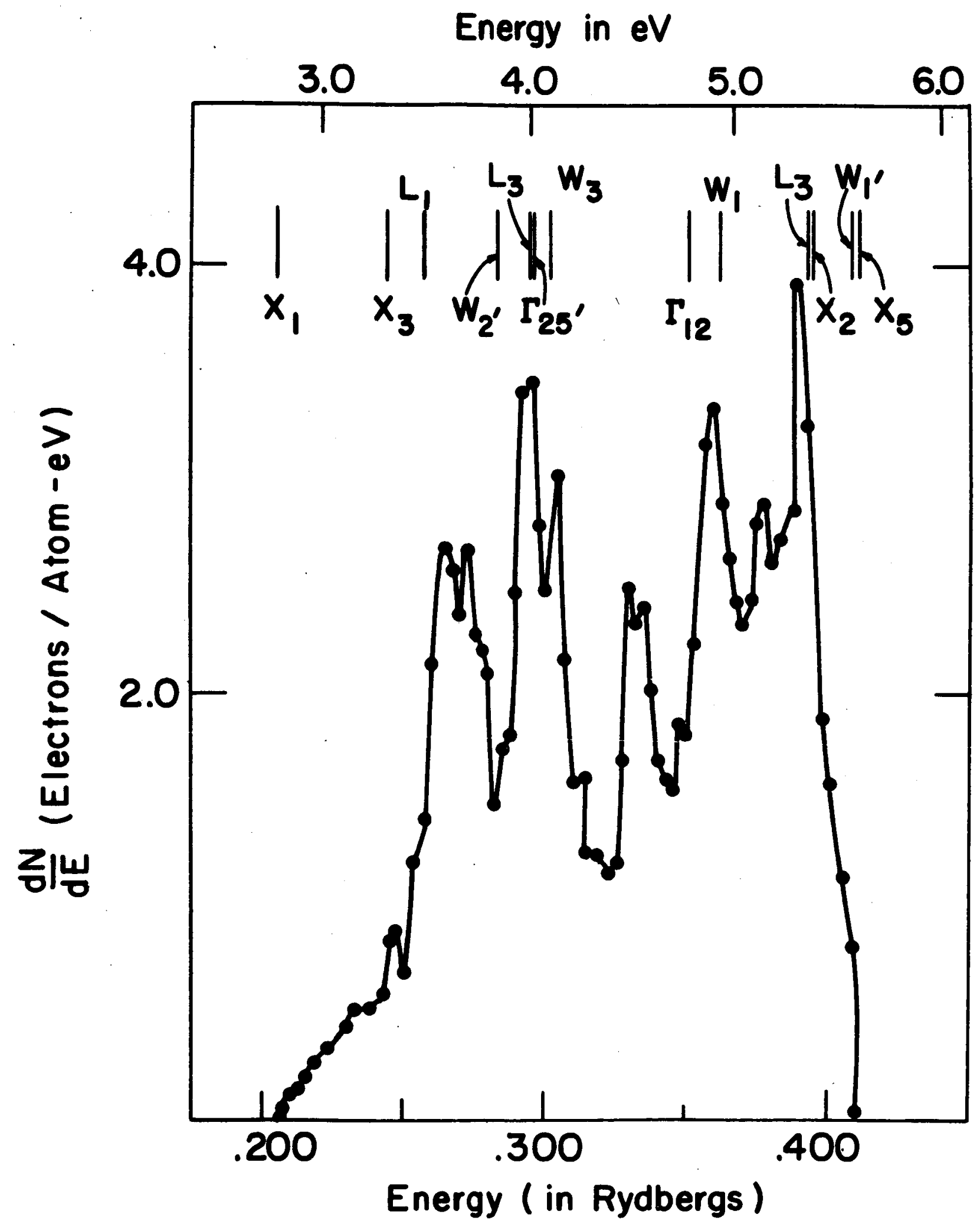

Fig. 5 


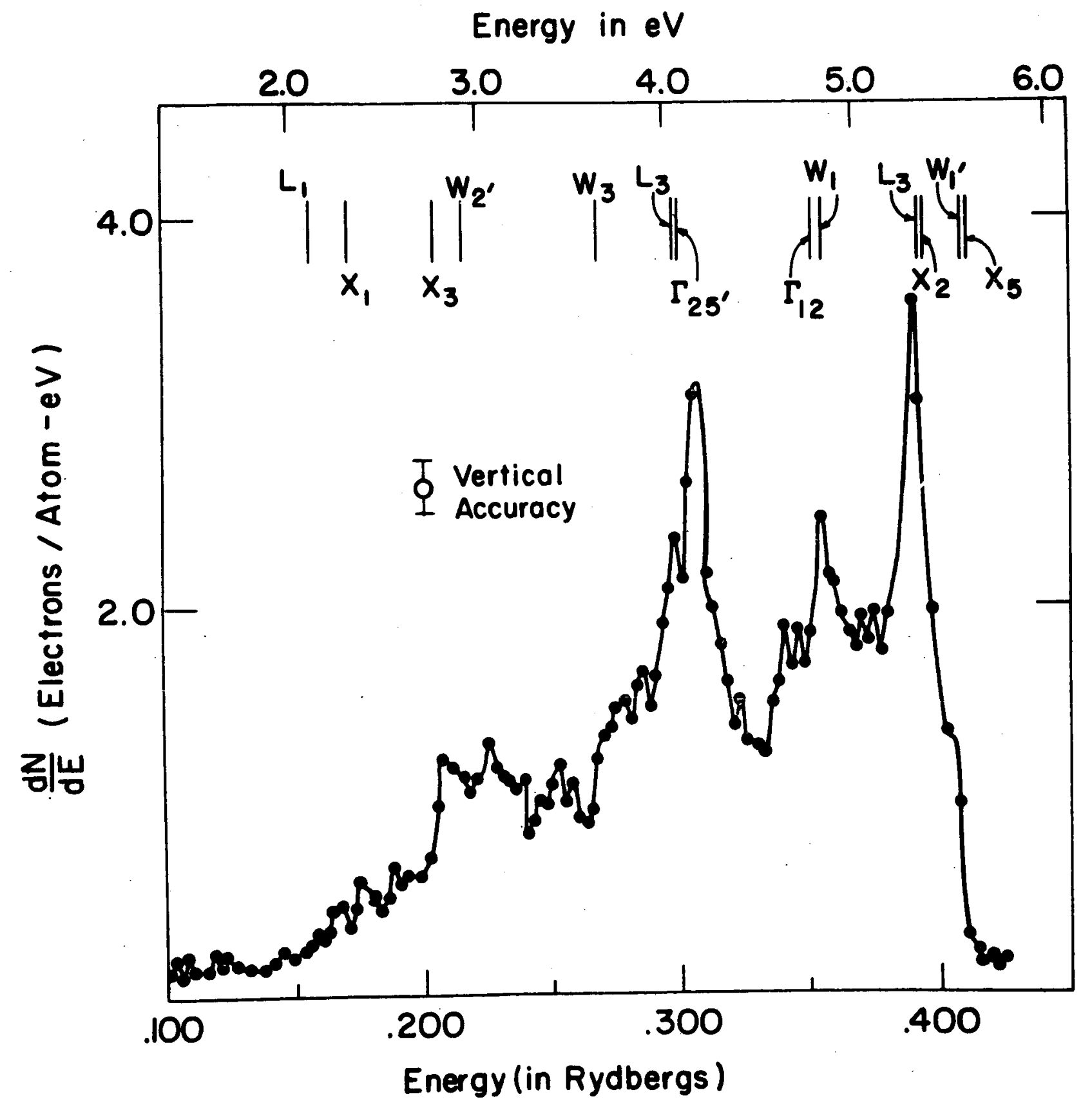

Fig. 6 


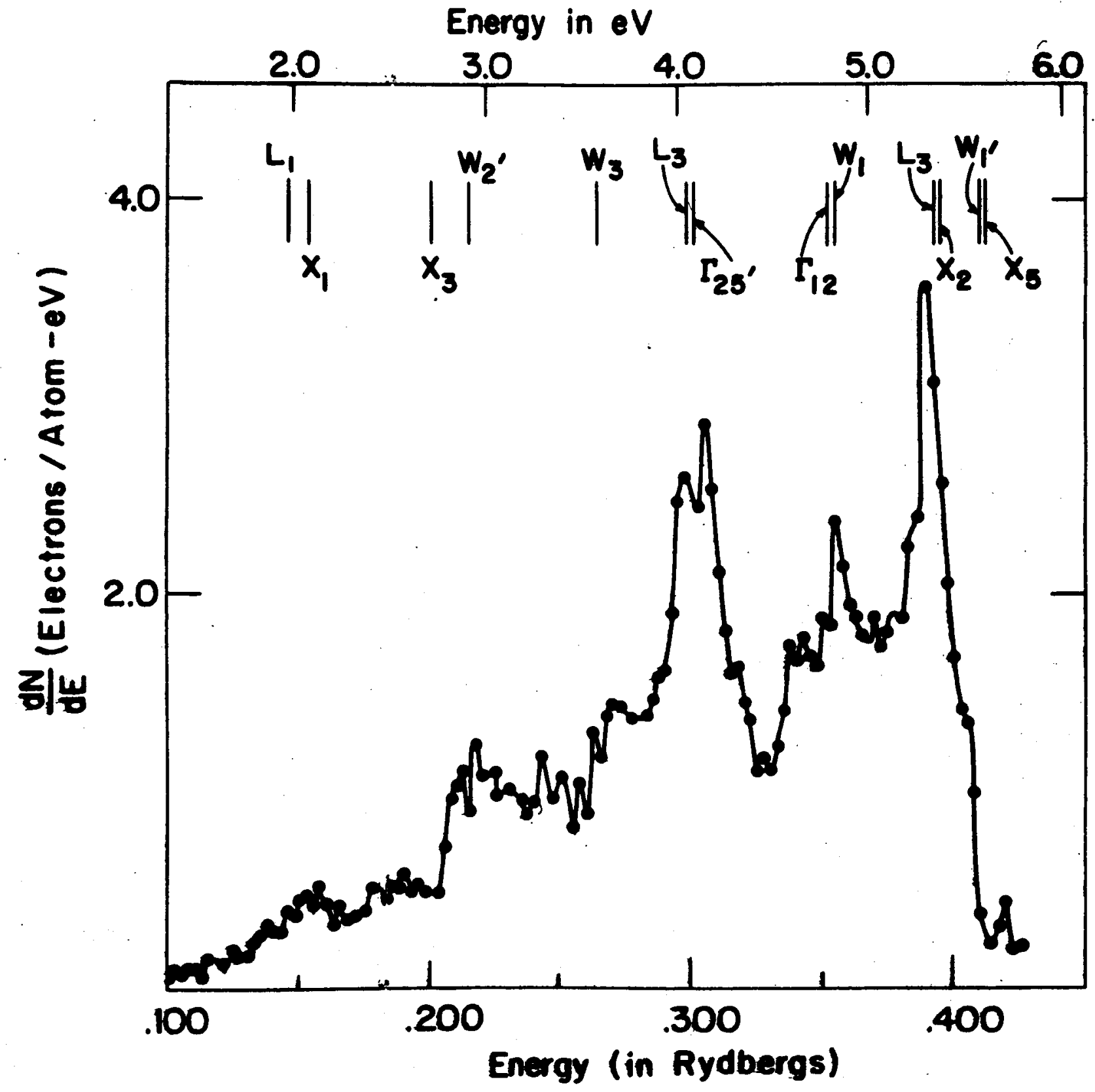

Fig. 7 


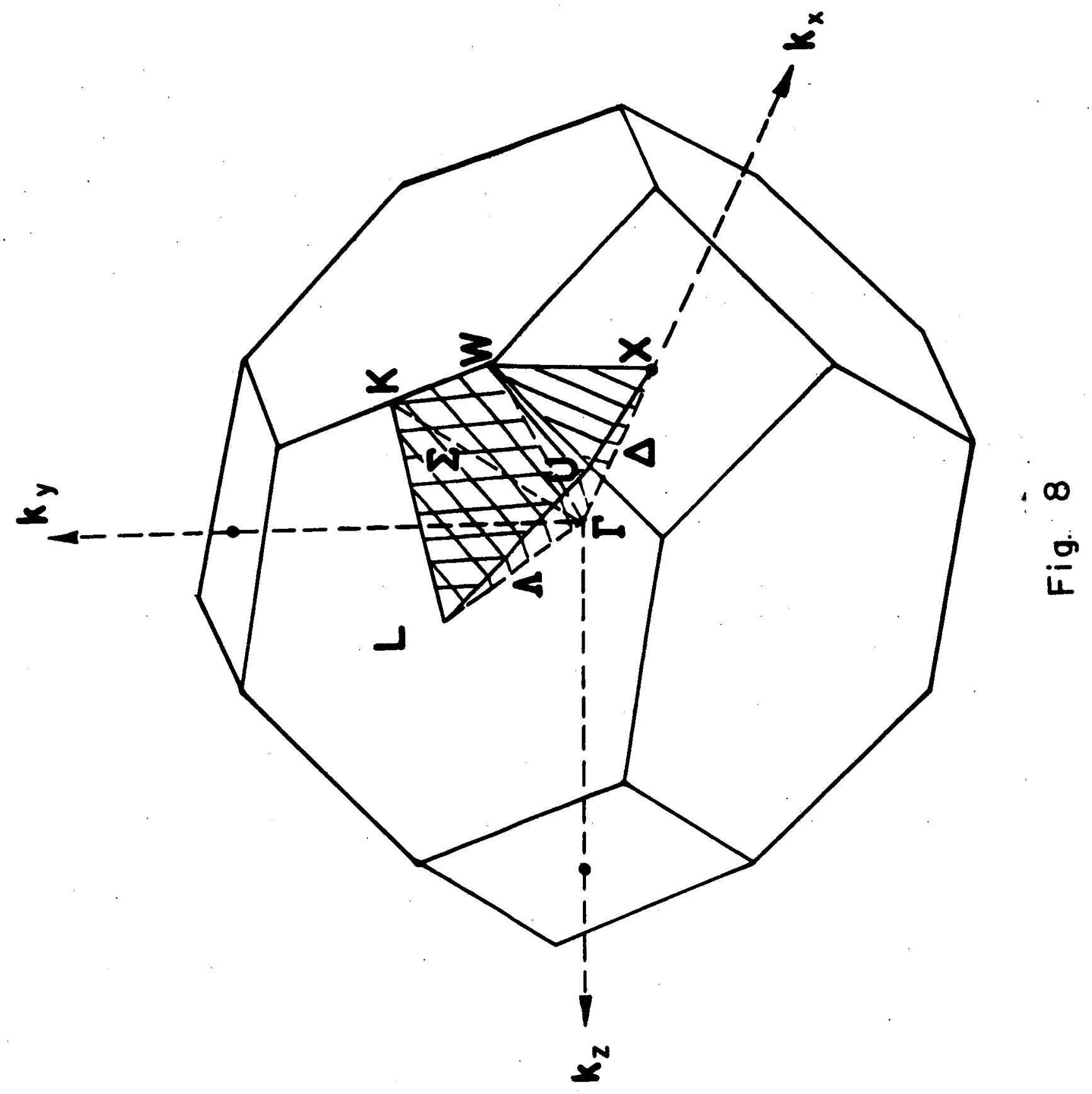

